\title{
Ammonium Ion Repression of Sporulation in Saccharomyces cerevisiae
}

\author{
By J. RICHARD DICKINSON* AND IAN W. DAWES \\ Department of Microbiology, University of Edinburgh, Edinburgh, EH9 3JG, U.K.
}

(Received 8 September 1982; revised 4 January 1983)

\begin{abstract}
The role of $\mathrm{NH}_{4}^{+}$in the regulation of sporulation in Saccharomyces cerevisiae has been studied by analysing the effects of $\mathrm{NH}_{4}^{+}$and methylammonium ions on sporulation in a wild-type strain; a strain homozygous for the spd1 mutation, (derepressed sporulation on a number of carbon sources, reduced sensitivity to $\mathrm{NH}_{4}^{+}$-inhibition of sporulation); and a strain homozygous for the gdhA6 mutation, (the structural gene for the anabolic $\mathrm{NADP}^{+}$-dependent glutamate dehydrogenase). The addition of ammonium or methylammonium ions to sporulation medium resulted in incomplete ascus formation. The gdhA6 mutation resulted in a loss of sensitivity to $\mathrm{NH}_{4}^{+}$-inhibition of initiation of sporulation. At higher concentrations of $\mathrm{NH}_{4}^{+}$the strain homozygous for the gdhA6 mutation was even less sensitive than the sporulation-derepressed strain. However, in the formation of complete asci all three strains behaved very similarly over the whole range of ammonium sulphate concentration. These studies indicate there are at least two separate stages affected by $\mathrm{NH}_{4}^{+}$; one early, possibly initiation, the other later, concerned with the organization and delimitation of mature spores. From the reduced sensitivity to $\mathrm{NH}_{4}^{+-}$ inhibition of initiation of sporulation conferred by the gdhA6 mutation, and the fact that similar results were obtained using methylammonium ion, it is concluded that some metabolite of $\mathrm{NH}_{4}^{+}$ is responsible rather than $\mathrm{NH}_{4}^{+}$itself. In addition, the data provide some insight into the nature of the spdl mutation.
\end{abstract}

\section{INTRODUCTION}

Diploid strains of Saccharomyces cerevisiae transferred from a rich growth medium into one containing a poorly utilized carbon source such as acetate (and usually in the absence of nitrogen) switch from vegetative growth and mitosis to the alternative developmental pathway of meiosis and sporulation. The role of nitrogen metabolism in the control of initiation of sporulation is far from clear, and there are conflicting reports in the literature. Sporulation is repressed by ammonium ions (Miller, 1963). The mechanism of this effect is unknown for there is a lack of agreement on whether $\mathrm{NH}_{4}^{+}$itself participates directly, or whether it is due to derivatives (e.g. glutamine) synthesized from it (Piñon, 1977; Dubois et al., 1977; DurieuTrautmann \& Delavier-Klutchko, 1977; Delavier-Klutchko et al., 1980). Ammonium ion and glutamine are reported to have at least two sites of action: one quite early preventing RNA, protein and glycogen synthesis, and affecting pre-meiotic DNA synthesis; the other later when DNA synthesis is completed (Durieu-Trautmann \& Delavier-Klutchko, 1977; DelavierKlutchko \& Durieu-Trautmann, 1978; Delavier-Klutchko et al., 1980; Piñon, 1977; Croes et al., 1978). $\mathrm{NH}_{4}^{+}$does not inhibit the initiation of pre-meiotic DNA synthesis (Piñon, 1977), but it is reported that DNA synthesis (post initiation) is arrested by $\mathrm{NH}_{4}^{+}$(Piñon, 1977), or at least reduced and slowed down by $\mathrm{NH}_{4}^{+}$or glutamine (Durieu-Trautmann \& Delavier-Klutchko, 1977). Other biochemical processes relevant to sporulation that are inhibited by $\mathrm{NH}_{4}^{+}$include the induction of glyoxylate cycle enzymes and adaptation to acetate (Gosling \& Duggan, 1971), and glycogen degradation (Fonzi et al., 1979).

One approach to understanding the role of $\mathrm{NH}_{4}^{+}$repression of sporulation has been through the study of mutants. Dawes (1975) has described spdI mutants of $S$. cerevisiae which are 
derepressed for sporulation; these homozygous diploids sporulate under certain conditions in which the wild-type does not. In addition, these mutants show a significantly decreased sensitivity to $\mathrm{NH}_{4}^{+}$repression of sporulation (Vezinhet et al., 1979). To test whether the anabolic $\mathrm{NAPD}^{+}$-dependent glutamate dehydrogenase [L-glutamate : $\mathrm{NADP}^{+}$oxidoreductase (deaminating) (EC 1.4.1.4)] is involved in the repression of sporulation by $\mathrm{NH}_{4}^{+}$, Newlon (1979) constructed a strain homozygous for the gene gdhA6 (a nonsense mutation in the structural gene for $\mathrm{NADP}^{+}$-dependent glutamate dehydrogenase (Grenson et al., 1974). He found that the presence of absence of NADP+-dependent glutamate dehydrogenase made little difference to the cells' sensitivity to $\mathrm{NH}_{4}^{+}$repression of sporulation. The present study has analysed the repression of sporulation in strains that are : homozygous for the $s p d 1$ mutation; homozygous for the gdhA6 mutation; and in the wild-type strain $\mathrm{S} 41$. This has revealed that $\mathrm{NH}_{4}^{+}$repression of sporulation is due to some metabolite that is derived via the $\mathrm{NADP}^{+}$-dependent glutamate dehydrogenase, and has also given some insights into the role of the SPD 1 gene in sporulation.

\section{METHODS}

Yeast strains. The wild-type strain S41 of Saccharomyces cerevisiae $\left(\frac{M A T \mathrm{a}}{M A T \alpha} \frac{H O}{H O} \frac{\arg 4-1}{\arg 4-1} \frac{c y h I}{c y h 1}\right)$ was originally obtained from H. O. Halvorson, Brandeis University, Waltham, Massachusetts, U.S.A. Strain 59.4A $\left(\frac{M A T \mathrm{a}}{M A T \alpha} \frac{H O}{H O} \frac{\arg 4-1}{\arg 4-1} \frac{\mathrm{cyhl}}{\mathrm{cyhl} l} \frac{\mathrm{spdl}-1}{\mathrm{spdl} l-1}\right)$ was derived from $\mathrm{S} 41$ by UV mutagenesis, selected by an ether selection technique (Dawes \& Hardie, 1974), and re-dissected to yield the homothallic diploid segregant. Strain 275.5D

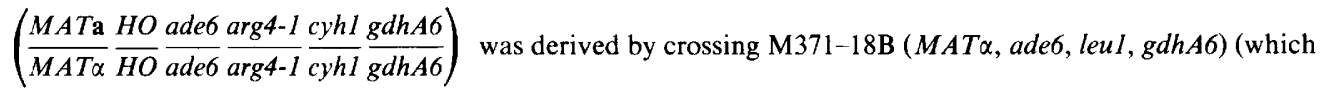
was obtained from T. Cooper, University of Pittsburgh, Pennsylvania, U.S.A.) with a haploid strain MATa, met l, ade 2 and repeated backcrossing of the haploid segregants which bore the gdhA6 allele to $\mathrm{S} 41$.

Culture media. Cells were grown with shaking at $30{ }^{\circ} \mathrm{C}$ in conical flasks filled to $40 \%$ total volume in a complex YEPD medium comprising (per litre): yeast extract $10 \mathrm{~g}$, bacteriological peptone $20 \mathrm{~g}$, glucose $20 \mathrm{~g}$, adenine $0 \cdot 2 \mathrm{~g}$, and uracil $0.2 \mathrm{~g}$. Sporulation medium contained (per litre): potassium acetate, $10 \mathrm{~g}$; adenine, $0.2 \mathrm{~g} ;$ uracil, $0.2 \mathrm{~g}$ and benzyl penicillin, $0 \cdot 15 \mathrm{~g}$. Strains were maintained on YEPD agar at $0{ }^{\circ} \mathrm{C}$ and were cloned before use.

Sporulation conditions. Cells were grown in the YEPD medium and harvested in early-stationary phase by centrifugation $\left(2000 \mathrm{~g}\right.$ for $10 \mathrm{~min}$ at $\left.30^{\circ} \mathrm{C}\right)$. The growth medium was discarded and the cells resuspended immediately in sporulation medium.

Counting procedures. The percentage of sporulation was estimated after $48 \mathrm{~h}$ by examination under the microscope of at least 300 cells. Viability was estimated by plating appropriate dilutions on YEPD agar plates and comparison with the results obtained using a Coulter Counter.

\section{RESULTS AND DISCUSSION}

Addition of ammonium or methylammonium ions to sporulation medium resulted in incomplete ascus formation, nevertheless it was clear that the cells had progressed a considerable way towards the formation of complete spores (Fig. 1). The typical morphology of these incomplete asci comprised ill-defined bundles of material. The spore coat material (which in a normal ascus appears exceptionally bright when viewed under phase-contrast) was largely absent. These incomplete asci retained the shape of vegetative cells. The spores contain haploid nuclei. Cells which had obviously initiated ascus formation were scored as such, as they were distinguishable from cells which had not initiated spore formation, and from those which had formed complete asci. Recognition of this effect has an important bearing on interpretation of the experimental results.

Figure 2 shows the effect of ammonium sulphate concentration on the ability of the three strains to initiate spore formation. It is clear that up to about $4 \mathrm{~mm}$-ammonium sulphate the 

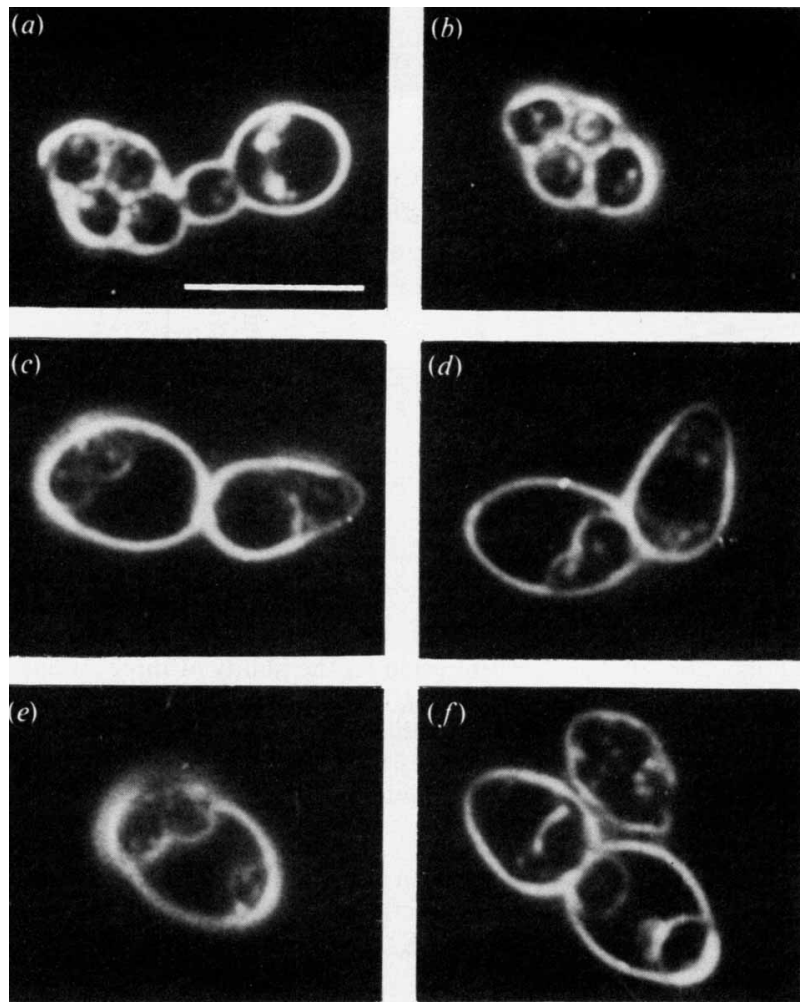

Fig. 1. Effect of $2 \mathrm{~mm}$-methylammonium chloride on sporulation of strain 275.5D. Dark-field illumination was used. Ascus walls and spore walls show up as brilliant white lines. The spore wall material in incomplete asci is largely absent and these spores are imperfectly defined. $(a, b)$ Normal four-spored asci; $(c-f)$ incomplete asci. The bar marker represents $10 \mu \mathrm{m}$ (the magnification is the same in all the photographs).

strain that is derepressed for sporulation (59.4A) was least affected by $\mathrm{NH}_{4}^{+}$. The homozygous gdhA6 strain fell roughly half way between 59.4A and the wild-type $\mathrm{S} 41$ in terms of its sensitivity to $\mathrm{NH}_{4}^{+}$. At concentrations of ammonium sulphate above $4 \mathrm{~mm}$ strain $275.5 \mathrm{D}$ is least sensitive to the ammonium ion. The wild-type is the most sensitive strain over the whole ammonium sulphate concentration range. This result is clearly different from that of Newlon (1979) who reported similar levels of sporulation in wild-type strains and in strains bearing the gdhA6 mutation. The reasons for the difference between these findings and those of Newlon (1979) are not obvious: it is possibly a consequence of pre-sporulation conditions, though one cannot be certain because Newlon (1979) did not describe his pre-sporulation conditions precisely. Perhaps the genetic background in which his gdhA6 strains were constructed did not allow clear expression of the later ammonium-inhibited stage described here. It is also possible that, unknown to Newlon, all of the strains that he used carried the spdl gene. We have on more than one occasion received strains from other laboratories which adventitiously bore an spdl allele.

Figure 3 shows the effect of ammonium sulphate concentration on the ability of the three strains to form complete asci. The results were quite different from those in Fig. 2, the three strains behaved very similarly over the whole range of ammonium sulphate concentration. Strain 275.5D showed some hypersensitivity to $\mathrm{NH}_{4}^{+}$at $1 \mathrm{mM}$-ammonium sulphate, although this detail was quite variable in replicate experiments. These results are very similar to those of Newlon (1979). It should be noted that the results here do not contradict those of Vezinhet et al. 


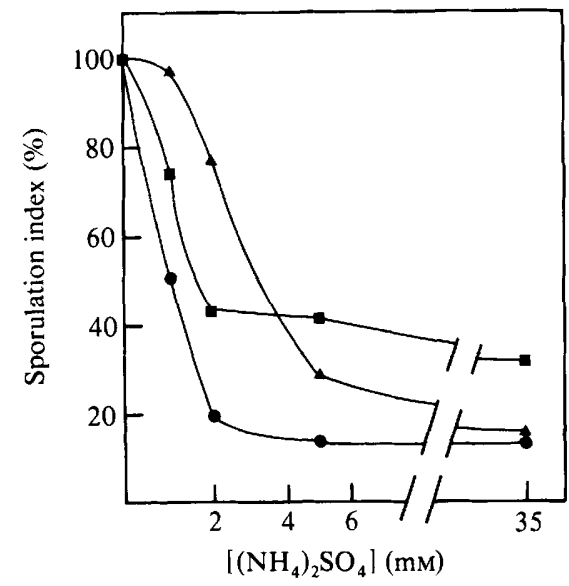

Fig. 2

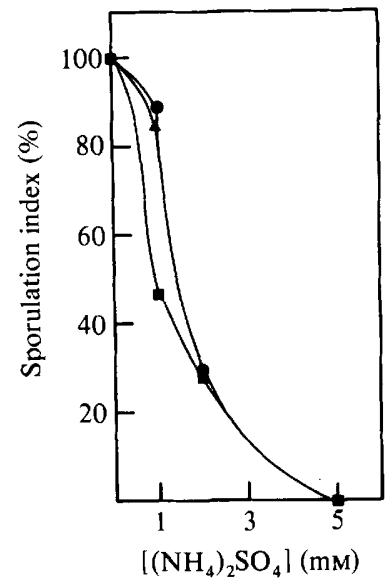

Fig. 3

Fig. 2. Effect of ammonium sulphate concentration on the ability of three strains of $S$. cerevisiae to initiate spore formation. Strains $\mathrm{S} 41(\mathbf{O}), 59.4 \mathrm{~A}(\boldsymbol{\Delta})$ and $275.5 \mathrm{D}(\square)$ were grown in a pre-sporulation YEPD medium, harvested in early-stationary phase and resuspended in sporulation medium as described in Methods. Sporulation index expresses the percentage sporulation relative to that which occurred for each strain in the absence of ammonium sulphate. All asci that had been initiated were scored.

Fig. 3. Effect of ammonium sulphate concentration on the ability of the three strains to form complete asci. The data shown relate to the experiment described in Fig. 2 except that only complete asci were

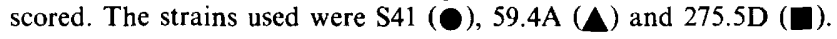

Table 1. Effect of ammonium sulphate concentration on the ability of the three strains to initiate and complete asci

The data shown relate to the experiment described in Fig. 2. The figures in parentheses denote the sporulation index which expresses the percentage sporulation (initiation, or formation of complete asci) relative to that which occurred for each strain in the absence of ammonium sulphate.

\begin{tabular}{|c|c|c|c|c|c|c|}
\hline \multirow[b]{2}{*}{$\begin{array}{l}\left(\mathrm{NH}_{4}\right)_{2} \mathrm{SO}_{4} \\
\text { concn in } \\
\text { sporulation } \\
\text { medium } \\
(\mathrm{mM})\end{array}$} & \multicolumn{2}{|c|}{ Strain S41 } & \multicolumn{2}{|c|}{ Strain $59.4 \mathrm{~A}$} & \multicolumn{2}{|c|}{ Strain $275.5 \mathrm{D}$} \\
\hline & $\begin{array}{l}\text { Absolute } \\
\text { percentage } \\
\text { of cells } \\
\text { initiating } \\
\text { spore } \\
\text { formation }\end{array}$ & $\begin{array}{l}\text { Absolute } \\
\text { percentage } \\
\text { of cells } \\
\text { forming } \\
\text { complete } \\
\text { asci }\end{array}$ & $\begin{array}{l}\text { Absolute } \\
\text { percentage } \\
\text { of cells } \\
\text { initiating } \\
\text { spore } \\
\text { formation }\end{array}$ & $\begin{array}{l}\text { Absolute } \\
\text { percentage } \\
\text { of cells } \\
\text { forming } \\
\text { complete } \\
\text { asci }\end{array}$ & $\begin{array}{l}\text { Absolute } \\
\text { percentage } \\
\text { of cells } \\
\text { initiating } \\
\text { spore } \\
\text { formation }\end{array}$ & $\begin{array}{l}\text { Absolute } \\
\text { percentage } \\
\text { of cells } \\
\text { forming } \\
\text { complete } \\
\text { asci }\end{array}$ \\
\hline 0 & $51(100)$ & $27(100)$ & $78(100)$ & $64(100)$ & $58(100)$ & $43(100)$ \\
\hline 1 & $26(51 \cdot 0)$ & $24(88.9)$ & $76(97.4)$ & $54(84.4)$ & $43(74 \cdot 1)$ & $20(46 \cdot 5)$ \\
\hline 2 & $10(19 \cdot 6)$ & $8(29 \cdot 6)$ & $60(76.9)$ & $19(29 \cdot 7)$ & $25(43)$ & $12(27.9)$ \\
\hline 5 & $7(13 \cdot 7)$ & $0(0)$ & $22(28 \cdot 2)$ & $0(0)$ & $24(41 \cdot 4)$ & $0(0)$ \\
\hline 35 & $7(13.7)$ & $0(0)$ & $12(15.4)$ & $0(0)$ & $18(31.0)$ & $0(0)$ \\
\hline
\end{tabular}

(1979) who reported that strains homozygous for spdl showed reduced sensitivity to ammonium ion repression of sporulation compared with the wild-type S41. Vezinhet et al. (1979) recorded the absolute level of sporulation, whereas the results presented in Figs 2 and 3 are the percentage sporulation relative to that which occurred for each strain in the absence of $\mathrm{NH}_{4}^{+}$. Strain 59.4A (homozygous for $s p d I$ ) sporulated far more extensively in absolute terms than the other strains used (Table 1). 


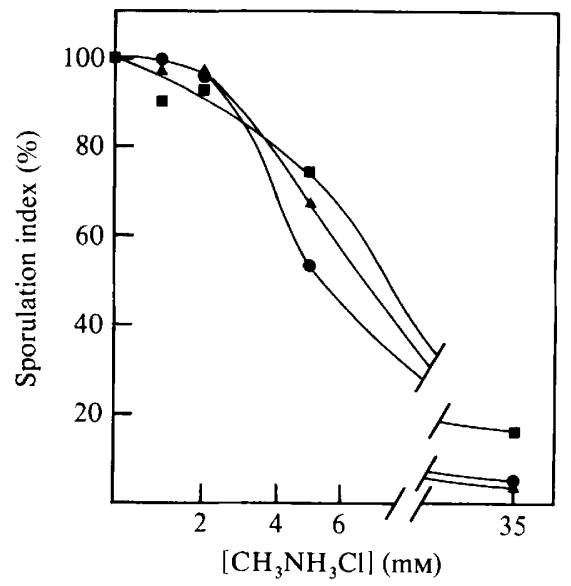

Fig. 4

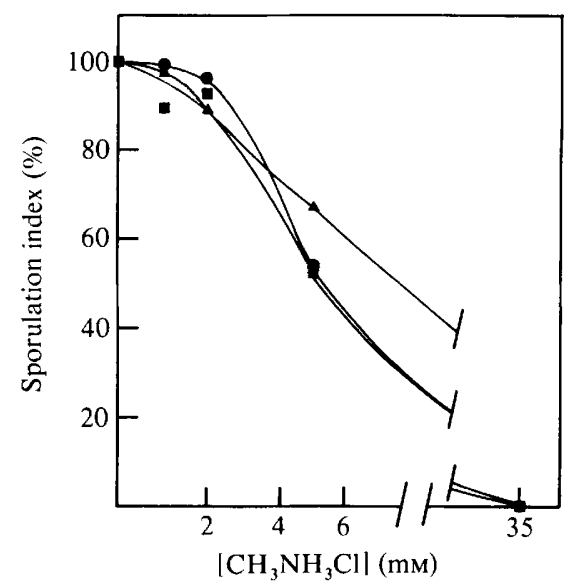

Fig. 5

Fig. 4. Effect of methylammonium chloride concentration on the ability of the three strains to initiate spore formation. Strains S41 (O), 59.4A $(\mathbf{A})$ and 275.5D $(\square)$ were grown in a pre-sporulation YEPD medium, harvested in early-stationary phase and resuspended in sporulation medium as described in Methods. Sporulation index expresses the percentage sporulation relative to that which occurred for each strain in the absence of methylammonium chloride. All asci that had been initiated were scored.

Fig. 5. Effect of methylammonium chloride concentration on the ability of the three strains to form complete asci. The data shown relate to the experiment described in Fig. 4 except that only complete asci were scored. The strains used were S41 (O), 59.4A (A) and 275.5D ( $\boldsymbol{\square})$.

Methylammonium ion gave similar results to ammonium ion (Figs 4 and 5). The fact that the strain lacking $\mathrm{NADP}^{+}$-dependent glutamate dehydrogenase was least sensitive to repression of initiation of sporulation by both ammonium ion and methylammonium ion at the higher concentrations used, and was less sensitive than the wild-type over the whole $\mathrm{NH}_{4}^{+}$ concentration range, implied that some metabolite derived from the ammonium ion, rather than the ion itself is the effector repressing sporulation.

This study has identified two distinct stages of sporulation that are affected by the presence of $\mathrm{NH}_{4}^{+}$in the sporulation medium: initiation, and a later stage associated with the organization and delimitation of spores. The fact that the strain homozygous for the spdl gene showed a similar $\mathrm{NH}_{4}^{+}$-sensitivity response to the wild-type with respect to the formation of complete asci, (Fig. 3), even though the culture of strain 59.4A had a higher absolute degree of sporulation (Table 1), shows that the strains differ only in their relative abilities to initiate sporulation. That there is a late event in sporulation associated with spore delimitation, which is inhibited by the presence of $\mathrm{NH}_{4}^{+}$, is in accord with the findings of Illingworth (1973).

The discovery that a strain homozygous for the gdhA6 mutation mimics, at least in part, the behaviour of the $s p d l$ homozygous diploid, suggests that the regulation of $\mathrm{NADP}^{+}$-dependent glutamate dehydrogenase may be altered in spdl mutants. This could arise either by an alteration in the enzyme itself, or the availability of its substrates, or the carbon catabolite control over its synthesis and/or activity. The first of these possibilities is unlikely since the enzyme has been found to be active under a range of conditions (J. R. Dickinson, unpublished data). In wild-type strains of $S$. cerevisiae $\mathrm{NADP}^{+}$-dependent glutamate dehydrogenase is inactivated by glucose starvation (Mazón, 1978; Mazón \& Hemmings, 1979) showing that the enzyme is subject to carbon catabolite control under vegetative conditions.

We are grateful to Professor J. M. Mitchison for the use of a Zeiss microscope and to Mr D. Roy for assistance with photography. The work was supported by the Science and Engineering Research Council. 


\section{REFERENCES}

Croes, A. F., Steijns, J. M., DE VRies, G. \& VAN Der PUTTE, T. M. (1978). Inhibition of meiosis in Saccharomyces cerevisiae by ammonium ions: interference of ammonia with protein metabolism. Planta 141, 205-209.

DAWES, I. W. (1975). Study of cell development using derepressed mutations. Nature, London 255, 707708.

DAWES, I. W. \& HARDIE, I. H. (1974). Selective killing of vegetative cells in sporulated yeast cultures by exposure to diethyl ether. Molecular and General Genetics 131, 281-289.

Delavier-Klutchko, C. \& Durieu-Trautmann, O. (1978). Effect of ammonia on glycogen metabolism during sporulation of Saccharomyces cerevisiae. Journal of General Microbiology 108, 169-171.

Delavier-KlutchKo, C., Durieu-Trautmann, O., Allemand, P. \& TAvlitzki, J. (1980). Assimilation of ammonia during sporogenesis of Saccharomyces cerevisiae: effect of ammonia and glutamine. Journal of General Microbiology 116, 143-148.

Dubois, E., Vissers, S., Grenson, M. \& Wiame, J-M. (1977). Glutamine and ammonia in nitrogen catabolite repression of Saccharomyces cerevisiae. Biochemical and Biophysical Research Communications 75, 233-239.

Durieu-Trautmann, O. \& Delavier-Klutchko, C. (1977). Effect of ammonia and glutamine on macromolecular synthesis and breakdown during sporulation of Saccharomyces cerevisiae. Biochemical and Biophysical Research Communications 79, 438-442.

Fonzi, W. A., Shanley, M. \& Opheim, D. J. (1979). Relationship of glycolytic intermediates, glycolytic enzymes, and ammonia to glycogen metabolism during sporulation in the yeast Saccharomyces cerevisiae. Journal of Bacteriology 137, 285-294.

Gosling, J. P. \& DugGan, P. F. (1971). Activities of tricarboxylic acid cycle enzymes, glyoxylate cycle enzymes and fructose diphosphatase in baker's yeast during adaptation to acetate oxidation. Journal of Bacteriology 106, 908-914.

Grenson, M., Dubois, E. \& Piotrowska, M. (1974). Ammonia assimilation in Saccharomyces cerevisiae as mediated by the two glutamate dehydrogenases. Evidence for the gdhA locus being a structural gene for the NADP-dependent glutamate dehydrogenase. Molecular and General Genetics 128, 73-85.

ILLINGWORTH, R. F. (1973). Lipid composition of Saccharomyces cerevisiae DCL740 in relation to morphogenesis of ascospores. Ph.D. thesis. University of Bath. Bath, U.K.

MAzón, M. J. (1978). Effect of glucose starvation on the nicotinamide adenine dinucleotide phosphatedependent glutamate dehydrogenase of yeast. Journal of Bacteriology 133, 780-785.

Mazón, M. J. \& Hemmings, B. A. (1979). Regulation of Saccharomyces cerevisiae nicotinamide adenine dinucleotide phosphate-dependent glutamate dehydrogenase by proteolysis during carbon starvation. Journal of Bacteriology 139, 686-689.

Miller, J. J. (1963). The metabolism of yeast sporulation. V. Stimulation and inhibition of sporulation and growth by nitrogen compounds. Canadian Journal of Microbiology 9, 259-277.

Newlon, M. C. (1979). NADP-specific glutamate dehydrogenase is not involved in repression of yeast sporulation by ammonia. Molecular and General Genetics 176, 297-300.

PIÑon, R. (1977). Effects of ammonium ions on sporulation of Saccharomyces cerevisiae. Experimental Cell Research 105, 367-378.

VezinheT, F., KinNaird, J. H. \& DaWes, I. W. (1979). The physiology of mutants derepressed for sporulation in Saccharomyces cerevisiae. Journal of General Microbiology 115, 391-402. 